\title{
TRAS EL RASTRO DEL VOTO ESTRATÉGICO EN EL PRIMER BALOTAJE DE ARGENTINA: ANÁLISIS COMPARATIVO DE LA ASOCIACIÓN ENTRE LOS RESULTADOS DE LA PRIMERA Y SEGUNDA VUELTA ELECTORAL A NIVEL MESA DE VOTACIÓN
}

\author{
Daniela Zacharias, ${ }^{1}$ Gilda Garibotti ${ }^{2}$ y Daiana $\mathrm{Neri}^{3}$
}

\section{Resumen}

En octubre de 2015 se realizaron las elecciones presidenciales argentinas. Ninguno de los candidatos consiguió la cantidad de votos suficiente para ser proclamado presidente, lo cual definió un escenario de balotaje entre los dos más votados. Por otro lado, más de la cuarta parte del electorado votó por un candidato que no alcanzó a pasar a la segunda vuelta. En este trabajo se investiga cómo este electorado, y el conformado por quienes votaron en blanco o cuyo voto fue anulado, votó en el primer balotaje realizado en Argentina. Mediante análisis de regresión lineal múltiple se estudia la asociación entre el voto en primera vuelta de este electorado y el voto por alguno de los dos candidatos en el balotaje. La unidad de análisis es la mesa de sufragio, la menor unidad de agregación posible. Los análisis se hacen a nivel nacional, regional y por nivel educativo.

Palabras clave: polarización - trasvase - regresión lineal - elecciones - balotaje - Argentina

Presidential elections in Argentina were celebrated in October, 2015. None of the candidates obtained the necessary amount of ballots to be proclaimed president, which triggered a ballotage

\footnotetext{
${ }^{1}$ Magíster en Estadística, Docente en Departamento de Estadística, Universidad Nacional del Comahue, Argentina. danielazach@gmail.com.

${ }^{2}$ Doctora en Estadística, Docente en Departamento de Estadística, Universidad Nacional del Comahue, Argentina. garibottig@comahue-conicet.gob.ar.

${ }^{3}$ Candidata a Doctora en Ciencias Políticas. Docente en Universidad Nacional del Comahue y Universidad Nacional de Río Negro, Argentina. neridaiana@ hotmail.com.
} 
process between the two candidates with the highest voting support. On the other hand, over $25 \%$ of the citizens had chosen a candidate that did not get enough votes to participate in the second electoral round. In this paper we investigate how these citizens, and those that voted blank or had their votes voided in the first round, voted in the first ballotage performed in Argentina. By performing a multiple linear regression analysis we studied the association between the first round vote with the vote for one of the ballotage candidates. The analysis has been performed at the polling station level, the smallest available unit of analysis. We analyzed the results at national and regional levels, and also by level of education.

Key words: polarization - ballot reorientation - multiple linear regression models - elections - ballotage - Argentina

\section{Introducción}

La realización de elecciones competitivas hace de los estados sistemas políticos democráticos. Las elecciones permiten ejercer la representación política, la creación de gobiernos y la construcción de legitimidad para tomar decisiones en la cúpula estatal. Es allí que los ciudadanos pueden elegir sus representantes, emitir sus preferencias políticas, elegir nuevos gobiernos, castigar a gobiernos en curso y evaluar el resultado de las políticas públicas. Por su parte, los partidos políticos pueden competir por el acceso al poder, institucionalizar los conflictos y dar cuenta de los proyectos políticos que representan.

Es en las instancias electivas del juego democrático que se toman las decisiones que simultáneamente son colectivas (es decir, son tomadas por varias personas) y públicas (afectan a varias personas) (Colomer, 2001). Ahora bien, estas elecciones ciudadanas están ordenadas por reglas de decisión que convierten los votos en espacios de poder, 
cargos ejecutivos y legislativos. Las instituciones electorales determinan la conversión de votos en escaños. Además, estas instituciones demarcan resultados posibles y construyen tipos de mayorías.

El balotaje o segunda vuelta es una de las reglas electorales que fuerza la construcción de mayorias absolutas en la toma de decisión ciudadana. Establece que, en caso de no producirse una mayoría absoluta en la elección, se realice una segunda votación entre las dos opciones (o candidaturas) más votadas. Así, al restringirse las opciones solamente a dos, se descuenta que una de ellas obtendrá más de la mitad de los sufragios. Este sistema tiene su origen en Francia y es actualmente utilizado en numerosas elecciones presidenciales de América Latina. En Argentina, como producto del denominado "Pacto de Olivos", se incorporó el balotaje al sistema electoral con la reforma constitucional de 1994. En esta reforma, además, se eliminó el Colegio Electoral y se dispuso la elección directa para todos los cargos ejecutivos y legislativos.

El mecanismo de segunda vuelta elegido introduce una serie de innovaciones que lo convierten en una rareza en todo el mundo. Los artículos 97 y 98 de la Constitución prevén la realización de un balotaje en dos situaciones: cuando ninguno de los candidatos se alza con el $45 \%$ de los votos en primera vuelta o ninguno supera el $40 \%$ con una diferencia de más del $10 \%$ respecto del que le sigue en cantidad de sufragios. Si ninguna de estas condiciones se cumple, se realiza una segunda vuelta, que se debe efectuar dentro de los 30 días siguientes contados a partir de la última elección. Participan las dos fórmulas a presidente y vicepresidente más votadas en primera instancia y resulta electa aquella que obtenga la mayor cantidad de votos afirmativos válidamente emitidos (artículo 151 de la Ley 24.444).

La Constitución de 1994 rigió las elecciones que tuvieron lugar desde entonces: 1995, 1999, 2003, 2007 y 2011. De todos esos comicios, el único que arrojó un claro escenario de balotaje fue el del 27 de abril de 2003, cuando la 
Alianza Frente por la Lealtad (Carlos Menem - Juan Carlos Romero) obtuvo el 24,45 \% y la Alianza Frente para la Victoria (Néstor Kirchner - Daniel Scioli) logró el 22,24 \%. En ese escenario, habría correspondido celebrar una segunda vuelta el 18 de mayo de 2003. Sin embargo, previendo un voto contrario, Carlos Menem desistió de presentarse, por lo que Néstor Kirchner fue proclamado presidente.

El 22 de noviembre de 2015 se realizó por primera vez una elección de segunda vuelta presidencial en Argentina. En las elecciones generales del 25 de octubre ninguna de las fórmulas obtuvo la cantidad de votos necesarios para resultar electa y ninguno de los candidatos a participar del balotaje desistió de hacerlo. El tercer domingo de noviembre, Daniel Scioli, de la Alianza Frente para la Victoria, y Mauricio Macri, de la Alianza Cambiemos (37,1 \% y 34,2 \% de los votos positivos de primera vuelta, respectivamente), se disputaron en un balotaje la preferencia popular para obtener el cargo de presidente argentino. En esa instancia, más de un cuarto del total de electores tuvo que reconsiderar su elección de octubre y elegir entre Daniel Scioli, Mauricio Macri o bien tomar la opción de votar en blanco o anular el voto.

En este trabajo buscamos explorar esta primera experiencia tratando de identificar cómo se comportaron en el balotaje los electores que en la primera vuelta votaron a otros candidatos, intentando distinguir algunos de los factores tenidos en cuenta por los electores en ocasión de la segunda vuelta. Para ello, hemos trabajado con los resultados oficiales de ambas elecciones (primera y segunda vuelta electoral) a nivel mesa de votación $\mathrm{y}$, utilizando modelos de regresión lineal múltiple (Zacharias, Sulmont \& Garibotti, 2015), estimamos el grado en el que la votación en la primera vuelta por Sergio Massa, Margarita Stolbizer, Nicolás del Caño y Alberto Rodríguez Saá se asoció al voto por Scioli o Macri en el balotaje. También investigamos si en los resultados hay características diferenciales a nivel regional y la posible influencia de variables sociodemográficas, como el nivel educativo. 


\section{Escena política}

En la etapa previa a la segunda vuelta, entre el 25 de octubre y el 22 de noviembre de 2015, distintos analistas políticos y medios de prensa expresaban sus opiniones sobre el posible comportamiento del electorado frente al balotaje. Dado que la diferencia porcentual entre los candidatos era menor al $3 \%$, las predicciones sobre el resultado del balotaje dependían decisivamente de las suposiciones realizadas sobre el comportamiento de las distintas bases electorales de los candidatos y partidos que no llegaron a la segunda vuelta. Incluso, se popularizaron aplicaciones para simular los posibles resultados del balotaje según fuera el trasvase de votos entre primera y segunda vuelta. ${ }^{4}$ En todos los potenciales escenarios, resultaba definitoria la decisión de aquellos que habian votado por Massa para presidente ya que este había conseguido el tercer lugar en primera vuelta, captando al $20 \%$ del electorado.

Una vez planteado el balotaje, la atención inicial se volcó sobre las definiciones y actitudes de los candidatos con relación a la "conducción" de sus votantes. Al respecto, el único candidato que se manifestó explícitamente fue Nicolás del Caño, del Frente de Izquierda y de los Trabajadores (FIT), a favor del voto en blanco. Esta decisión fue acompañada con una fuerte campaña en medios y redes sociales. Del Caño señalaba que esta postura era una "primera medida de resistencia a los ajustes que aplicarán, apenas asuman, tanto Macri como Scioli" (La Izquierda Diario, 2015).

El resto de los candidatos -Massa, Stolbizer y Rodríguez Saá- no dieron un apoyo explícito a ninguno de los candidatos. Podría decirse que, en buena medida, los dos primeros lo hicieron en forma elíptica al apoyar "el cambio" (en relación al Frente Cambiemos de Macri) o a través de varias declaraciones en las que se mostraron en contra de la figura de Scioli como presidente del país (Diario La Nación, 2015b), (Radio 40, 2015). Massa sí fue explícito al indicar

\footnotetext{
${ }^{4}$ Ver, por ejemplo: http://lucianoamor.github.io/balotaje2015/
} 
a sus votantes que no deberían votar en blanco. "No voten en blanco, aún [sic] a riesgo de equivocarse. En la vida hay que jugarse" (Infobae, 2015), expresó. En el caso de Rodríguez Saá, se conjeturaba un posible apoyo a Scioli, que finalmente no se concretó.

Las encuestas realizadas en esta etapa del proceso electoral concordaban en que la mayoría de los votantes de Massa se inclinarían por $\mathrm{Macri}^{5}$. Según la encuesta de Poliarquía Consultores (Diario La Nación, 2015c) realizada entre el 9 y 10 de noviembre, los votos de los candidatos de la primera vuelta se repartirían tal como se muestra en la Tabla 1:

\section{Tabla 1}

\section{Encuesta de Poliarquía Consultores sobre el trasvase de votos}

Los votantes de los candidatos de la primera vuelta, ¿a quién elegirian?

\begin{tabular}{|l|c|c|c|c|c|}
\hline $\begin{array}{l}\text { Candidato por el que } \\
\text { votó en primera vuelta }\end{array}$ & M. Macri & D. Scioli & $\begin{array}{c}\text { Blanco/ } \\
\text { Anulado }\end{array}$ & Indecisos & Total \\
\hline M. Macri & $97 \%$ & $2 \%$ & $0 \%$ & $1 \%$ & $100 \%$ \\
\hline D.scioli & $3 \%$ & $95 \%$ & $0 \%$ & $2 \%$ & $100 \%$ \\
\hline S. Massa & $59 \%$ & $22 \%$ & $8 \%$ & $11 \%$ & $100 \%$ \\
\hline N. del Caño & $29 \%$ & $30 \%$ & $35 \%$ & $6 \%$ & $100 \%$ \\
\hline M. Stolbizer & $48 \%$ & $18 \%$ & $24 \%$ & $10 \%$ & $100 \%$ \\
\hline A. Rodríguez Saá & $28 \%$ & $39 \%$ & $18 \%$ & $15 \%$ & $100 \%$ \\
\hline
\end{tabular}

Fuente: Encuesta Poliarquia 11/11/2015 - La Nación (Diario La Nación, 2015c).

Ante la falta de definiciones concretas en los dias previos al balotaje, distintos actores políticos especularon sobre cómo se daría el trasvase de votos. Por ejemplo, Massa declaró en un programa televisivo que "hay una enorme mayoría de los que nos votaron que van a votar a Macri. Pero también hay un grupo muy importante que son laburantes, clase media, que tienen miedo al ajuste, que van a votar a

${ }^{5} 59 \%$ según Poliarquia, 50 \% según Haime y asociados, ambas de principios de noviembre. 
Scioli. Y algunos, una porción muy chiquitita, va a votar en blanco". ${ }^{6}$ Estas declaraciones tuvieron una enorme repercusión en los medios de prensa. Al mismo tiempo, otro dirigente del espacio de Massa, Felipe Solá, declaró que "como peronista le resulta muy difícil votar a Macri" (Diario Página $12,2015)$. Esta definición contribuyó al debate sobre si los votantes de Massa y Rodríguez Saá provenientes del peronismo podian elegir a un candidato no-peronista como Macri o no (Diario Clarín, 2015) (Diario Clarín, 2015b) (Diario La Nación, 2015).

Otros análisis, realizados antes y después del triunfo de Macri en el balotaje, buscaron predecir o explicar los resultados en base a parámetros socioeconómicos o regionales (INFO News, 2015) (Diario El Cronista Comercial, 2015).

\section{Datos y metodología}

Los datos utilizados en este estudio son los resultados provisorios de los escrutinios de las elecciones presidenciales de 2015, en primera y segunda vueltas, a nivel de mesa de sufragio en todo el país. Se excluyeron del análisis las mesas de sufragio en las que el total de votos positivos era igual a cero en alguna de las dos elecciones (1925 mesas) y en las que los votos emitidos representaban menos del $30 \%$ de los electores (115 mesas). Como resultado de estas consideraciones, el universo de estudio comprendió 96.752 mesas de sufragio.

Estudiamos la asociación entre la distribución de los votos en la primera y segunda vuelta por medio de modelos de regresión lineal múltiple. Las variables dependientes consideradas son:

-Diferencia de porcentajes de votos a favor de Mauricio Macri entre la segunda y la primera vuelta.

-Diferencia de porcentajes de votos a favor de Daniel Scioli entre la segunda y la primera vuelta. Las variables independientes fueron los porcentajes de votos obtenidos en la primera vuelta por los otros cuatro can-

${ }^{6} \mathrm{https}: / /$ www.youtube.com/watch?v=p0-6zlpCXuU. 
didatos participantes de la primera vuelta electoral -Sergio Massa, de Unidos por una Nueva Alternativa (UNA); Nicolás del Caño, del Frente de Izquierda y de los Trabajadores (FIT); Margarita Stolbizer, de Progresistas y Alberto Rodríguez Saá, de Compromiso Federal-y el porcentaje de votos no positivos: el conjunto de los votos blancos y los nulos.

Todos los porcentajes considerados son tomados sobre la base del total de votos emitidos en las respectivas vueltas electorales.

Los modelos considerados se expresan de la siguiente manera:

$M_{i}=\beta_{M, S M} S M_{i}+\beta_{M, N D C} N D C_{i}+\beta_{M, M S} M S_{i}+\beta_{M, A R S} A R S_{i}+\beta_{M, N P} N P_{i}+\varepsilon_{M, i}$

$S_{i}=\beta_{S, S M} S M_{i}+\beta_{S, N D C} N D C_{i}+\beta_{S, M S} M S_{i}+\beta_{S, A R S} A R S_{i}+\beta_{S, N P} N P_{i}+\varepsilon_{S, i}$

Donde:

$i=$ mesa de votación

$M_{i}=$ Diferencia (en \% de votos emitidos) de la votación por Macri

entre la segunda vuelta y la primera vuelta

$S_{i}=$ Diferencia (en \% de votos emitidos) de la votación por Scioli

entre la segunda vuelta y la primera vuelta

$S M_{i}=\%$ de votos emitidos obtenidos por Massa en primera vuelta

$N D C_{i}=\%$ de votos emitidos obtenidos por Del Caño en primera vuelta

$M S_{i}=\%$ de votos emitidos obtenidos por Stolbizer en primera vuelta

$A R S_{i}=\%$ de votos emitidos obtenidos por Rodríguez Saá en primera vuelta

$N P_{i}=\%$ de votos emitidos de votos No Positivos (blancos y nulos)

en primera vuelta

$\varepsilon_{M, i}$ y $\varepsilon_{S, i}$ corresponden al término de error aleatorio para cada uno de los modelos.

$\beta_{M, S M,} \beta_{M, N D C,} \beta_{M, M S} \beta_{M, A R S} \beta_{M, N P \text { son los coeficientes lineales para las va- }}$ riables independientes $S M_{i^{\prime}} N D C i, M S_{i^{\prime}} A R S_{i}$ y $N P_{i}$ en la variación de \% de votos de Macri.

$\beta_{S, S M} \beta_{S, N D C,} \beta_{S, M S}, \beta_{S, A R S}, \beta_{S, N P \text { son los coeficientes lineales para las variables }}$ independientes $S M_{i^{\prime}} N D C_{i^{\prime}} M S_{i^{\prime}} A R S_{i}$ y $N P_{i}$ en la variación de \% de votos de Scioli.

De aquí en adelante, con el solo fin de hacer más fácil la lectura de este documento, a menos que se especifique lo contrario, nos referiremos al "porcentaje de votos emitidos" como el "porcentaje de votos". Estos modelos se estimaron de manera global para las 96.752 mesas de votación consideradas y por regiones geográficas y categorías de nivel educativo. 
Las regiones geográficas consideradas son: Ciudad $\mathrm{Au}-$ tónoma de Buenos Aires (CABA), la provincia de Buenos Aires, Centro (provincias de Córdoba, Entre Ríos y Santa Fe), Noreste (provincias de Chaco, Corrientes, Formosa y Misiones), Noroeste (provincias de Catamarca, Jujuy, Salta, Santiago del Estero y Tucumán), Cuyo (provincias de La Rioja, Mendoza, San Juan y San Luis) y Patagonia (provincias de Chubut, La Pampa, Neuquén, Río Negro, Santa Cruz y Tierra del Fuego). Este criterio de división regional es usual en Argentina, dadas las características sociales, demográficas y económicas especificas de cada área.

Además del aspecto regional, resulta de interés en el análisis de estos modelos de trasvase de votos entre la primera vuelta y el balotaje tomar en cuenta variables que reflejen diferencias socioeconómicas. Para representar esta dimensión, y en base a los datos disponibles, hemos escogido un índice que refleja el nivel educativo (IE). El IE se toma en base al promedio de los años de estudio de los mayores de 25 años de cada hogar. Para su cálculo se tomaron los datos oficiales del Censo Nacional 2010. ${ }^{7}$ A cada mesa de sufragio se le asignó el IE promedio correspondiente al radio censal al que pertenece. Cabe destacar que los dos procesos electorales que abarca este estudio son de corte "circuital", es decir, los electores fueron agrupados por proximidad de su domicilio a la mesa de votación. Esto permite considerar el IE promedio del radio censal como un buen diferenciador/reflejo del nivel socioeconómico (NSE) de los votantes de la mesa. Las fuentes de datos utilizadas fueron tres:

- Resultados de los escrutinios provisorios de las Elecciones Presidenciales Argentinas 2015, a nivel mesa de sufragio. Datos obtenidos de la Dirección Nacional Electoral (DINE), Ministerio de Justicia y Derechos Humanos, Argentina.

- Ubicación de los recintos de votación a nivel nacional. Datos obtenidos del sitio Dat.Ar. ${ }^{8}$

\footnotetext{
${ }^{7}$ http://www.indec.gov.ar/.

${ }^{8} \mathrm{http}: / /$ datar.noip.me/dataset/establecimientos-electorales-paso-2015.
} 
-Datos georreferenciados del Censo Nacional de Población, Hogares y Viviendas 2010 - Instituto Nacional de Estadística y Censos (INDEC), a nivel radio censal.

El tratamiento georreferenciado de datos se realizó mediante el programa de código abierto QGIS v2.12 (Quantum GIS Development Team, 2015). Para el análisis estadístico se utilizó el paquete estadístico R v. 3.2.2 (R Core Team, 2015).

\section{Resultados}

\section{Resultados generales}

La Tabla 2 muestra los resultados de la primera vuelta de la elección para presidente de 2015 en Argentina. Para cada candidato se presenta la cantidad de votos obtenidos y los porcentajes sobre votos positivos y emitidos.

Tabla 2

Resultados de la primera vuelta de las Elecciones Presidenciales Argentina 2015

\begin{tabular}{|c|c|c|c|c|}
\hline ORGANIZACIÓN POLÍTICA & $\begin{array}{l}\text { Candidato } \\
\text { presidencial }\end{array}$ & Votos & $\begin{array}{l}\text { \% sobre } \\
\text { votos } \\
\text { positivos }\end{array}$ & $\begin{array}{c}\text { \% sobre } \\
\text { votos } \\
\text { emitidos }\end{array}$ \\
\hline $\begin{array}{l}\text { Alianza Frente para } \\
\text { la Victoria }\end{array}$ & Daniel Scioli & 9.338 .490 & $37,1 \%$ & $35,9 \%$ \\
\hline Alianza Cambiemos & Mauricio Macri & 8.601 .131 & $34,2 \%$ & $33,0 \%$ \\
\hline $\begin{array}{l}\text { Alianza Unidos por una } \\
\text { nueva alternativa (una) }\end{array}$ & Sergio Massa & 5.386 .977 & $21,4 \%$ & $20,7 \%$ \\
\hline $\begin{array}{l}\text { Alianza Frente de Izquierda } \\
\text { y de los Trabajadores }\end{array}$ & Nicolás del Caño & 812.530 & $3,2 \%$ & $3,1 \%$ \\
\hline Alianza Progresista & Margarita Stolbizer & 632.551 & $2,5 \%$ & $2,4 \%$ \\
\hline $\begin{array}{l}\text { Alianza Compromiso } \\
\text { Federal }\end{array}$ & $\begin{array}{l}\text { Adolfo Rodríguez } \\
\text { Saá }\end{array}$ & 412.578 & $1,6 \%$ & $1,6 \%$ \\
\hline & Votos positivos & 25.184 .257 & $100,0 \%$ & $97,5 \%$ \\
\hline & Votos blancos & 664.740 & & $2,6 \%$ \\
\hline & Votos nulos & 199.449 & & $0,8 \%$ \\
\hline \multicolumn{2}{|c|}{ Total de votos emitidos } & 26.048 .446 & & $100,0 \%$ \\
\hline \multicolumn{2}{|c|}{ Total de electores hábiles } & 32.130 .853 & Participación: & $80,8 \%$ \\
\hline
\end{tabular}

Fuente: DINE, Min. de Justicia y Derechos Humanos, Argentina. ${ }^{9}$

\footnotetext{
${ }^{9} \mathrm{http}: / /$ elecciones.gob.ar/articulo_princ.php?secc=2\&sub_secc=55.
} 
Los resultados de la segunda vuelta electoral se presentan en la Tabla 3.

Tabla 3

Resultados del Balotaje Presidencial Argentina 2015

\begin{tabular}{|l|l|c|c|c|}
\hline ORGANIZACIÓN POLÍTICA & $\begin{array}{l}\text { Candidato } \\
\text { presidencial }\end{array}$ & Votos & $\begin{array}{c}\% \text { sobre votos } \\
\text { positivos }\end{array}$ & $\begin{array}{c}\% \text { sobre votos } \\
\text { emitidos }\end{array}$ \\
\hline Alianza Cambiemos & Mauricio Macri & 12.997 .937 & $51,3 \%$ & $50,1 \%$ \\
\hline $\begin{array}{l}\text { Alianza Frente } \\
\text { para la Victoria }\end{array}$ & Daniel Scioli & 12.317 .330 & $48,7 \%$ & $47,5 \%$ \\
\hline Votos positivos & 25.315 .267 & $100,0 \%$ & $97,5 \%$ \\
\hline Votos blancos & 306.651 & & $1.2 \%$ \\
\hline Votos nulos & 330.998 & & $1,3 \%$ \\
\hline & Total de votos emitidos & 25.952 .906 & & $100,0 \%$ \\
\hline \multicolumn{2}{r|r}{ Total de electores hábiles } & 32.130 .853 & Participación: & $80,8 \%$ \\
\hline
\end{tabular}

Fuente: DINE, Min. de Justicia y Derechos Humanos, Argentina. ${ }^{10}$

Entre la primera vuelta electoral y el balotaje, el porcentaje de votos para Daniel Scioli aumentó del 35,9 \% al 47,5 \%, mientras que el de Mauricio Macri creció del 33,0 \% al 50,1 \%. Se observa que el incremento porcentual de votos a favor de Macri superó al recibido por Scioli en más de cinco puntos, suficiente para darle el triunfo por un margen estrecho. Esta situación, en la que el presidente electo no fue el candidato más votado en primera vuelta, es, de acuerdo con lo publicado por el politólogo Ignacio Labaquí, relativamente inusual (Berensztein, 2015) (Bastión Digital, 2015). De los 42 balotajes previos a este realizados en América Latina desde la recuperación democrática de los años setenta, este caso de reversión de voto de primera vuelta solamente se verificó en 11 oportunidades. En la Figura 1 se presenta la distribución del incremento en el porcentaje de votos para cada uno de los candidatos por mesa de votación. Se observa que, mientras que la mediana de crecimiento de Macri es de unos 17 puntos porcentuales por mesa, la de Scioli es de solamente 12 puntos. Incluso el límite del tercer cuartil de Scioli se encuentra por debajo de la mediana de Macri.

${ }^{10} \mathrm{http}$ ://elecciones.gob.ar/articulo_princ.php?secc=2\&sub_secc=57. 


\section{Figura 1}

Diagrama de cajas de la distribución de la diferencia de porcentajes de votos entre vueltas electorales, por mesa de sufragio (Segunda vuelta - Primera vuelta)

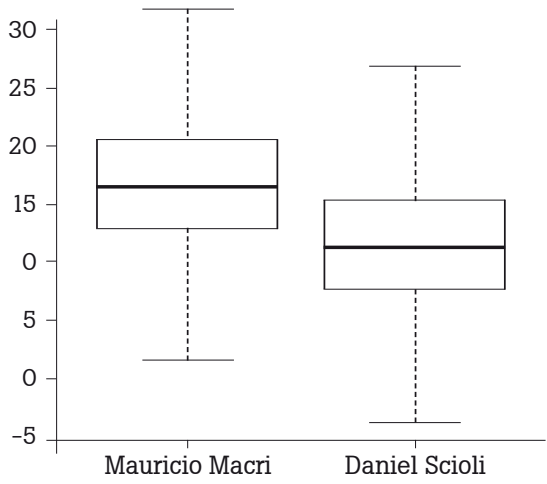

Para realizar el ajuste de los modelos representados por las ecuaciones (1) y (2) aplicamos el método de regresión lineal múltiple utilizando los datos individuales por mesa. De esa forma, obtuvimos los coeficientes que se presentan en la Tabla 4.

Tabla 4

Coeficientes de modelos de regresión lineal de trasvase de votos

\begin{tabular}{|c|c|c|}
\hline & \multicolumn{2}{|c|}{ Variable dependiente } \\
\hline Coeficientes* $^{*}$ & $M$ & $S$ \\
\hline$\beta_{S M}$ & 0,63 & 0,34 \\
\hline$\beta_{N D C}$ & 0,13 & 0,67 \\
\hline$\beta_{M S}$ & 0,45 & 0,23 \\
\hline$\beta_{A R S}$ & 0,62 & 0,35 \\
\hline$\beta_{N P}$ & 0,42 & 0,48 \\
\hline$R^{2}$ ajustado & 0,93 & 0,87 \\
\hline
\end{tabular}

*Todos los coeficientes son significativos, $\mathrm{p}<0,001$.

Según estos resultados, cada punto en el porcentaje de votos obtenidos por Massa en la primera vuelta se asocia a un crecimiento de 0,63 en la diferencia de porcentajes de vo- 
tos recibidos por Macri entre el balotaje y la primera vuelta, mientras que dicho crecimiento es de solo 0,34 para Scioli. Se observa un comportamiento similar cuando se analiza la contribución del voto de Rodríguez Saá hacia los votos de los candidatos participantes en el balotaje. El electorado de Stolbizer favorece en mayor medida a Macri que a Scioli, dado que el aumento de un punto en el porcentaje de votos obtenidos por Stolbizer produciría un aumento de 0,45 en la diferencia entre segunda y primera vuelta en el porcentaje de votos de Macri y de 0,23 en el de Scioli. En cambio, el porcentaje de votos obtenido por Del Caño favorece a Scioli. Por cada punto de aumento en el porcentaje de votos a Del Caño en octubre, aumenta en 0,67 la diferencia de votos de Scioli entre vueltas y tan solo en 0,13 la correspondiente a Macri.

El 22 de noviembre los electores tuvieron otra alternativa de elección: realizar un voto "no positivo", esto es, optar por votar en blanco o anular el voto. En un modelo de regresión lineal múltiple similar a los dos anteriores, pero considerando como variable dependiente el porcentaje de votos no positivos en cada mesa de sufragio, se obtuvieron los resultados que figuran en la Tabla 5.

Tabla 5

Coeficientes de modelos de regresión lineal para votos no positivos

\begin{tabular}{|c|c|}
\hline & Variable dependiente \\
\hline Coeficientes* $^{*}$ & NP2 \\
\hline$\beta_{S M}$ & 0,03 \\
\hline$\beta_{N D C}$ & 0,20 \\
\hline$\beta_{M S}$ & 0,31 \\
\hline$\beta_{A R S}$ & 0,03 \\
\hline$\beta_{N P}$ & 0,10 \\
\hline$R^{2}$ ajustado & 0,71 \\
\hline
\end{tabular}

*Todos los coeficientes son significativos, $\mathrm{p}<0,001$.

La mayor incidencia relativa sobre el voto no positivo corresponde a los votantes de Stolbizer (0,31). Resulta curioso 
que dicha incidencia fuese mayor que la de los votantes de Del Caño, quien llamó explícitamente a sus votantes a expresarse de esta manera. Los resultados de la Tablas 4 y 5 se presentan en forma gráfica en la Figura 2.

Figura 2

Coeficientes de regresión de los modelos aplicados

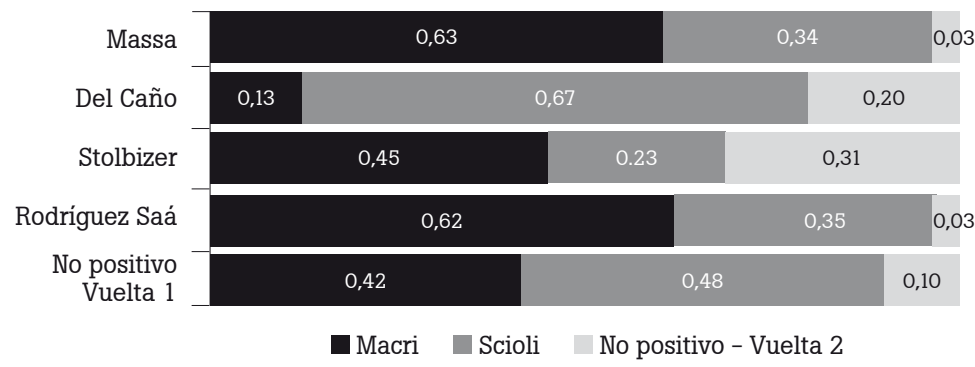

Análisis por región

La Tabla 6 presenta los promedios de los porcentajes de votos a nivel de mesa electoral para la primera y la segunda vuelta electoral por región.

Tabla 6

Promedio de porcentajes de votos en las mesas de sufragio de cada región

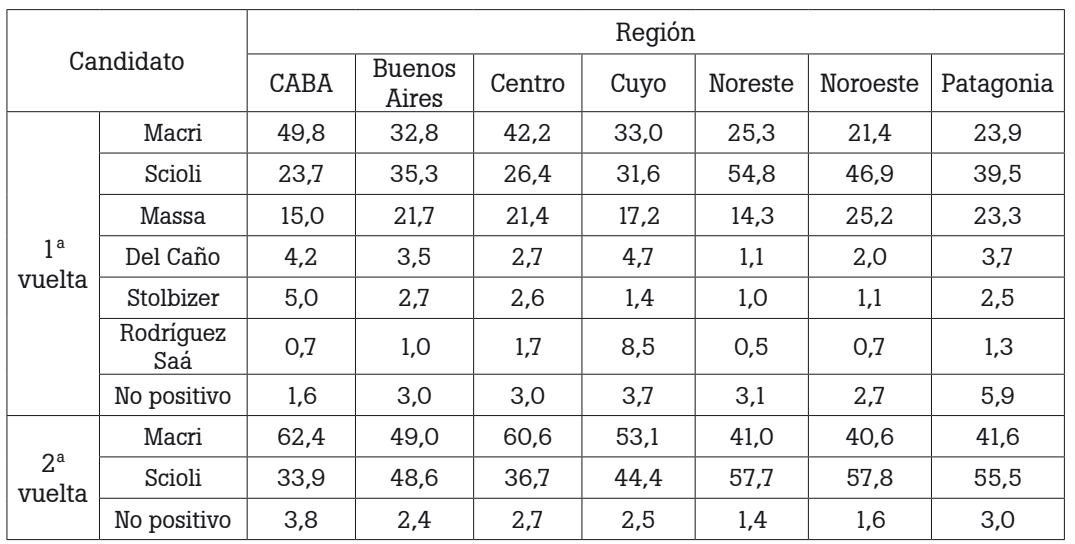


Se observa que en ambas vueltas electorales Macri se impuso en la Ciudad Autónoma de Buenos Aires y la región Centro. El Noreste y el Noroeste, así como la Patagonia, fueron bastiones de Scioli. En la provincia de Buenos Aires y en Cuyo obtuvieron Macri y Scioli porcentajes de votos similares en la primera vuelta. El virtual empate se mantuvo solo en la provincia de Buenos Aires en el balotaje, puesto que en esta segunda elección, en la región de Cuyo, el porcentaje de votos de Macri superó al de Scioli por casi $10 \%$. En el resto de las regiones, los mayores porcentajes corresponden a Scioli. Se observa que en regiones donde Scioli hizo una muy buena primera vuelta (Noreste y Noroeste), a pesar de obtener un triunfo holgado en el balotaje, las diferencias porcentuales entre balotaje y primera vuelta favorecen ampliamente a Macri. Así, por ejemplo, en el Noreste, mientras que Scioli creció 2,9 \% entre primera vuelta y balotaje, Macri lo hizo 15,7\%. Solamente en CABA, la provincia de Buenos Aires y la Patagonia, los crecimientos de Macri fueron muy similares a los de Scioli. Estos resultados se resumen en la Tabla 7, donde se muestra que las regiones de mayor crecimiento de Macri con respecto a su contrincante, Scioli, fueron las de Cuyo en primer lugar, con el 20,2 \%, Noroeste con el 19,3 \% y Centro con el 18,4\%. Cabe notar que en todas las regiones Macri obtuvo un mayor crecimiento en el porcentaje de votos que Scioli, incluso en aquellas en donde este candidato había mostrado ser preferido por una gran parte del electorado. Scioli tuvo su peor crecimiento en el Noreste, donde tan solo logró aumentar el 2,9 \%.

Tabla 7

Diferencia entre porcentajes de votos emitidos entre vueltas por región

\begin{tabular}{|c|c|c|c|c|c|c|c|}
\hline $\begin{array}{c}\text { Diferencia } \\
\text { entre } \\
\text { porcentajes }\end{array}$ & CABA & $\begin{array}{c}\text { Buenos } \\
\text { Aires }\end{array}$ & Centro & Cuyo & Noreste & Noroeste & Patagonia \\
\hline Macri & 12,6 & 16,1 & 18,4 & 20,2 & 15,7 & 19,3 & 17,7 \\
\hline Scioli & 10,2 & 13,3 & 10,3 & 12,7 & 2,9 & 10,9 & 16,0 \\
\hline Macri-Scioli & 2,4 & 2,8 & 8,1 & 7,5 & 12,8 & 8,4 & 1,7 \\
\hline
\end{tabular}


Para comprender el impacto que estas diferencias regionales tienen a nivel global es necesario tomar en cuenta el peso que estas tienen en cuanto a la población electoral. En la Figura 3 se puede observar que más del $40 \%$ de los votos emitidos corresponden a la región de Buenos Aires, que, junto con $\mathrm{CABA}$, representa aproximadamente la mitad de los electores del país. Las regiones del Noreste, Noroeste, Cuyo y Patagonia, en su conjunto, corresponden a una tercera parte del total.

Figura 3

Distribución de los votos emitidos por región

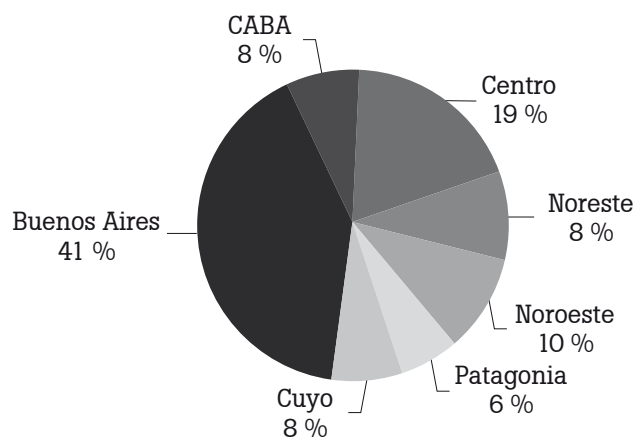

Los coeficientes de ajuste lineal de los modelos representados por las ecuaciones (1) y (2) aplicados por regiones son los que muestran las Figuras 4 y 5, respectivamente. De manera general, se observa un importante trasvase de votos de Massa hacia Macri, superando coeficientes de 0,5 en todas las regiones geográficas. Una fuente importante de votos adicionales para Macri se ubica en quienes eligieron inicialmente a Stolbizer, en todas las regiones exceptuando el Noreste. Es notorio que los coeficientes de regresión son muy cercanos a la unidad para el trasvase de votos que obtuvo Rodríguez Saá hacia Macri, sobre todo en la provincia de Buenos Aires, CABA y Centro. Solo los votantes de Del Caño mostraron una preferencia mayor hacia Scioli con respecto a Macri en la votación del balotaje en todas las regiones, con excepción del Noroeste. 


\section{Figura 4 \\ Coeficientes de regresión para el Modelo (1), según región"1"}

Variable dependiente:

Diferencia en \% de votos obtenidos por MACRI entre la segunda y la primera vuelta

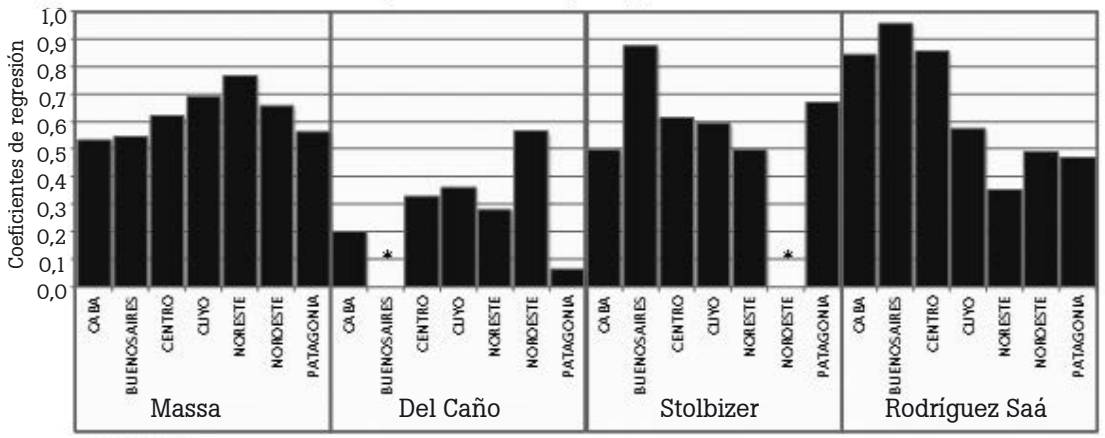

*No significativo

\section{Figura 5}

Coeficientes de regresión para el Modelo (2), según región ${ }^{12}$

Variable dependiente:

Diferencia en $\%$ de votos obtenidos por SCIOLI entre la segunda y la primera vuelta

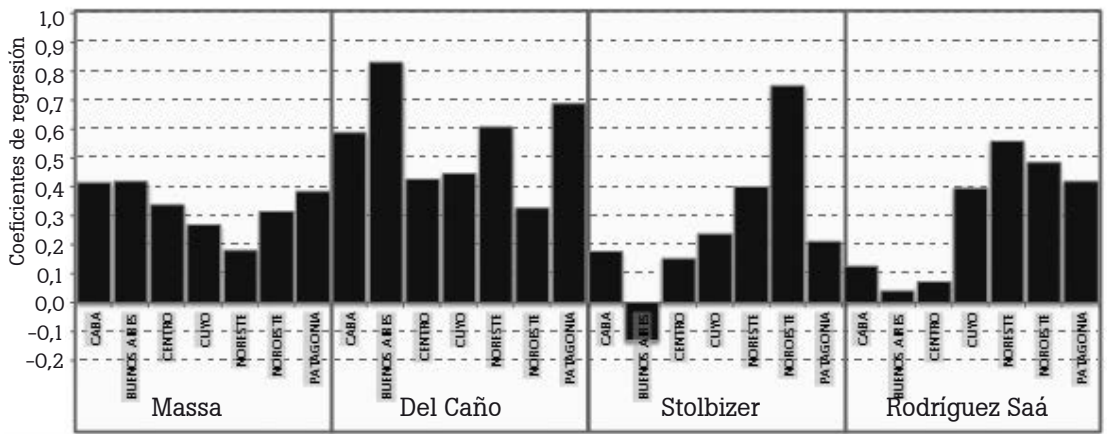

*No significativo

${ }^{11}$ Coeficientes de regresión para los votos no positivos: 0,2 en la región Cuyo; 0,3 en Buenos Aires, Centro y Patagonia; 0,4 en CABA; 0,5 en la región Noroeste y 1,1 en Noreste. El $\mathrm{R}^{2}$ ajustado de los modelos es mayor a 0,9.

${ }^{12}$ Coeficientes de regresión para los votos no positivos: -0,2 en la región Noreste; 0,4 en CABA y Noroeste; 0,5 en el Centro; 0,6 en Buenos Aires y la Patagonia y 0,7 en la región Cuyo. Los $\mathrm{R}^{2}$ ajustados de los modelos son mayores a 0,8 , con excepción del correspondiente a la región Noreste, que presenta un $\mathrm{R}^{2}$ ajustado de 0,33. 


\section{Análisis según Î́ndice de Educación (IE)}

La Tabla 8 presenta los promedios de los porcentajes de votos a nivel de mesa electoral para la primera y la segunda vuelta electoral por quintil de Índice de Educación (IE). El Quintil 1 corresponde al de menor IE y el Quintil 5 al de mayor IE.

Tabla 8

Promedio de porcentajes de votos en las mesas de sufragio en cada quintil de IE

\begin{tabular}{|c|c|c|c|c|c|c|}
\hline \multirow{2}{*}{\multicolumn{2}{|c|}{ Candidato }} & \multicolumn{5}{c|}{ Índice de Educación } \\
\cline { 2 - 7 } & Quintil 1 & Quintil 2 & Quintil 3 & Quintil 4 & Quintil 5 \\
\hline \multirow{4}{*}{} & Macri & 24,5 & 29,4 & 32,3 & 36,3 & 46,8 \\
\cline { 2 - 7 } & Scioli & 47,5 & 37,6 & 34,9 & 31,2 & 25,1 \\
\cline { 2 - 7 } $1^{\text {a }}$ vuelta & Massa & 18,8 & 22,8 & 22,4 & 21,8 & 17,3 \\
\cline { 2 - 7 } & Del Caño & 2,2 & 3,0 & 3,0 & 3,4 & 3,7 \\
\cline { 2 - 7 } & Stolbizer & 1,4 & 1,9 & 2,2 & 2,7 & 3,9 \\
\cline { 2 - 7 } & Rodriguez Saá & 1,9 & 1,8 & 1,9 & 1,6 & 1,0 \\
\cline { 2 - 7 } & No positivo & 3,6 & 3,5 & 3,3 & 3,0 & 2,2 \\
\hline \multirow{3}{*}{$\begin{array}{c}2^{\text {a }} \\
\text { vuelta }\end{array}$} & Macri & 40,0 & 47,4 & 50,7 & 54,8 & 62,2 \\
\cline { 2 - 7 } & Scioli & 58,1 & 50,4 & 46,9 & 42,6 & 34,8 \\
\cline { 2 - 7 } & No positivo & 1,8 & 2,3 & 2,4 & 2,6 & 3,0 \\
\hline
\end{tabular}

Tanto en la primera como en la segunda vuelta se observa que el voto por Macri aumenta en relación directa al nivel educativo del electorado. En el caso de la votación por Scioli la relación es inversa. Estas relaciones se pueden visualizar en la Figura 6. 
Figura 6

Porcentajes de votación para Macri y para Scioli según vuelta electoral y por quintil de educación

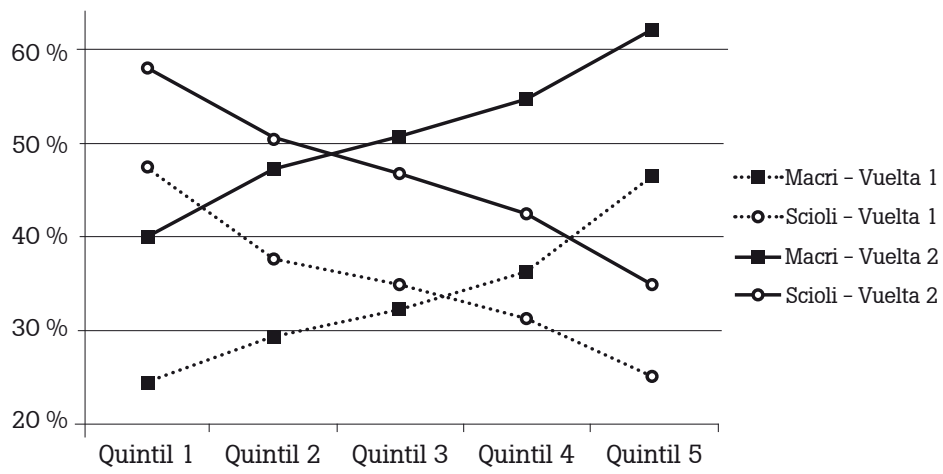

La Tabla 9 presenta las variaciones de los porcentajes de votos para Macri y Scioli entre vueltas en función del IE. Se observa que el comportamiento es similar en todos los niveles, con un crecimiento porcentual considerablemente mayor para Macri en todo el espectro.

Tabla 9

Diferencia entre porcentajes de votos emitidos entre vueltas por quintil de IE

\begin{tabular}{|c|c|c|c|c|c|}
\hline \multirow{2}{*}{$\begin{array}{c}\text { Diferencia entre } \\
\text { porcentajes }\end{array}$} & \multicolumn{5}{|c|}{ Índice de Educación } \\
\cline { 2 - 6 } & Quintil 1 & Quintil 2 & Quintil 3 & Quintil 4 & Quintil 5 \\
\hline Macri & 15,6 & 17,9 & 18,4 & 18,5 & 15,4 \\
\hline Scioli & 10,6 & 12,8 & 12,0 & 11,4 & 9,7 \\
\hline Macri-Scioli & 5,0 & 5,2 & 6,4 & 7,0 & 5,7 \\
\hline
\end{tabular}

En las Figuras 7 y 8 se representan los coeficientes de ajuste lineal del Modelo (1) y del Modelo (2), respectivamente, según el quintil de IE. Se puede observar, por un lado, que el comportamiento de quienes votaron a Massa es relati- 
vamente uniforme para todo el espectro social en relación al apoyo a Macri. Además, el peso de los electores de Rodríguez Saá en el aumento del porcentaje de votos obtenidos por Macri entre la segunda y la primera vuelta también es alto, y aumenta de manera significativa en las mesas con electores pertenecientes al cuarto y quinto superior en cuanto a IE.

El caudal de votos de Del Caño, derivado a cada uno de los candidatos del balotaje, muestra una tendencia conforme aumenta el nivel educativo (positiva en lo que respecta a Macri y negativa en el apoyo a Scioli) con punto de quiebre en el caso del quintil de mayor IE. Sin embargo, las diferencias son favorables a Scioli en todos estos casos.

Figura 7

Coeficientes de regresión para el Modelo (1), según quintil de $\mathrm{IE}^{13}$

Variable dependiente:

Diferencia en \% de votos obtenidos por MACRI entre la segunda y la primera vuelta

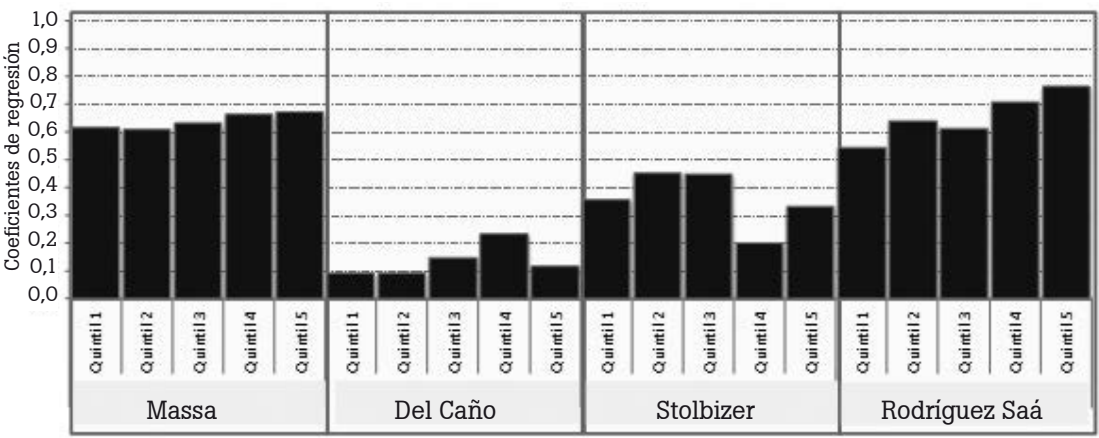

*No significativo

${ }^{13}$ Coeficientes de regresión para los votos no positivos: en los tres primeros quintiles: 0,4, en los dos últimos quintiles: 0,5. El $\mathrm{R}^{2}$ ajustado de los modelos son mayores a 0,88. 
Figura 8. Coeficientes de regresión para el Modelo (2), según quintil de $\mathrm{IE}^{14}$

Variable dependiente:

Diferencia en \% de votos obtenidos por SCIOLI entre la segunda y la primera vuelta

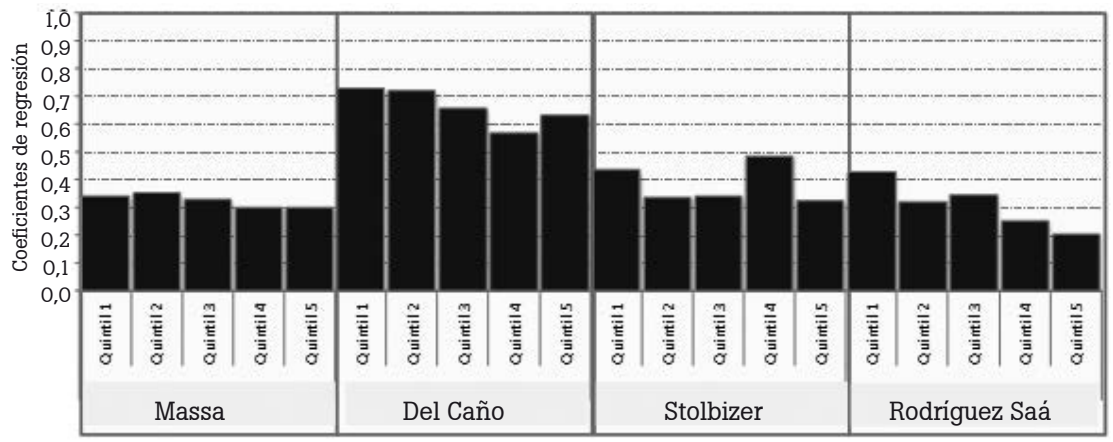

*No significativo

\section{Discusión}

Si bien los resultados de una sola elección no permiten extraer conclusiones sobre las trayectorias de los electores, el análisis de los datos permite reconocer varias tendencias y dar una primera respuesta a las preguntas que motivaron este estudio. Así, los resultados abren la posibilidad de observar cómo se comportaron en el balotaje los electores que en la primera vuelta votaron a otros candidatos, para poder identificar de manera preliminar qué factores tuvieron en cuenta los electores en ocasión del balotaje.

Respecto del primer interrogante, encontramos que a nivel global los votos de Sergio Massa resultaron decisivos para inclinar la balanza de la elección presidencial. En la segunda vuelta, el aporte de estos votantes se dividió en una fracción de 0,63 a 0,34 a favor de Mauricio Macri frente a Daniel Scioli, respectivamente. Puede decirse que prácticamente 2 de cada 3 votos originales de Massa se tradujeron

${ }^{14}$ Coeficientes de regresión para los votos no positivos: 0,$5 ; 0,4 ; 0,5 ; 0,4$ y 0,3 para los quintiles $1,2,3,4$ y 5, respectivamente. Los $\mathrm{R}^{2}$ ajustados de los modelos son mayores a 0,81 . 
en votos para Macri (Tabla 4 y Figura 2). La misma tendencia se observó en los votantes de Alberto Rodríguez Saá. También fue efectiva la sugerencia de Massa respecto a "jugarse" por un candidato, dado que fue muy bajo el porcentaje de votantes que optaron por hacerlo en blanco en el balotaje.

Los votantes de Nicolás del Caño en una gran mayoría desoyeron el mandato de voto en blanco del candidato y optaron por Scioli en el balotaje en una gran proporción $(0,67)$. Solamente una proporción cercana al $15 \%$ acató la indicación. Fue considerablemente mayor la incidencia de voto en blanco entre quienes optaron por Margarita Stolbizer, alrededor del $30 \%$.

Utilizando los coeficientes calculados con los Modelos (1) y (2) para repartir los votos de primera vuelta de cada uno de los candidatos hacia los candidatos del balotaje, es posible realizar una estimación de la composición del voto de la segunda vuelta electoral. El resultado de dicha estimación se presenta en la Figura 9. Puede verse que la victoria de Macri se sustenta en 2/3 de votos que podríamos llamar propios, mientras que el tercio restante corresponde al aporte de otros partidos, en particular a Massa. En este sentido, la base electoral de Scioli parece ser más firme, dado que $3 / 4$ partes de sus votos son propios.

Figura 9

Composición estimada del voto de Macri y Scioli en el balotaje

Composición estimada del voto de Macri

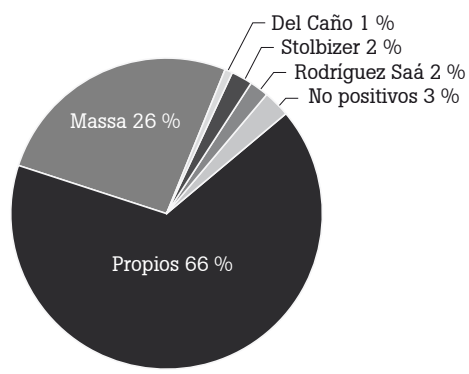

Composición estimada del voto de Scioli

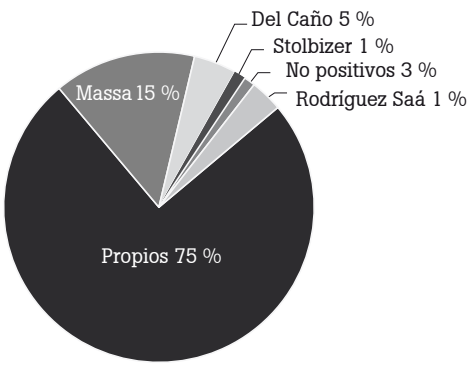


En el análisis del comportamiento a nivel regional, Macri recibió un importante aporte de votos de Massa, Rodríguez Saá y Stolbizer en las regiones en las que había mostrado mayor debilidad durante la primera vuelta. Si bien estos votos adicionales no le alcanzaron para ganar a nivel región, la diferencia porcentual lo favoreció claramente en el resultado global (Tabla 7) debido a que mantuvo el caudal electoral en sus bastiones (CABA y Centro) entre la primera vuelta y el balotaje. Este aporte se magnifica si se tiene en cuenta el exiguo margen por el cual se impuso finalmente, que fue inferior al $3 \%$ de los votos positivos.

Ahora bien, a través de nuestro segundo interrogante buscamos acercarnos a cuáles son algunos de los determinantes del voto de los candidatos de este balotaje a partir de las respuestas obtenidas en el análisis de trasvase. Las motivaciones detrás del voto han generado un gran volumen de literatura. ${ }^{15}$ No obstante, no es el objetivo de este trabajo abordar esa discusión sino comenzar a explorar algunos factores. Uno de los enfoques clásicos para indagar en los determinantes del voto es el llamado sociológico, el cual tiene como una de las variables explicativas más importantes a la clase social. En este trabajo abordamos los indicadores socioeconómicos de clase a partir del comportamiento por Índice Educativo. Al respecto, se pudo observar que el apoyo a Macri fue aumentando a medida que aumenta el IE. Por el contrario para Scioli la tendencia se invierte y encuentra que su mayor franja de apoyos está asociada a las preferencias de los votantes con menor nivel de IE. Podemos afirmar que hay una marcada tendencia de las clases más populares a inclinarse por el Frente para la Victoria mientras que los sectores de clases más altas optaron por la Alianza Cambiemos.

Por otro lado, como mencionamos previamente, los votantes de Massa y Rodríguez Saá se comportaron de mane-

${ }^{15}$ Para ver información al respecto se recomienda ver Lago, Montero \& Torcal, 2007; y Ratto \& Montero, 2013. 
ra similar y resultaron decisivos al triunfo de Macri. Cuando observamos cómo impacta el IE en los electorados de ambos candidatos, encontramos que en Massa no tuvo incidencia la pertenencia de clase en su preferencia en el balotaje. Esto puede explicar que, a pesar del mayor apoyo a Macri en el quintil de mayor IE, su crecimiento porcentual global en este segmento fue muy similar al correspondiente a los niveles de menor IE. En el caso de los votantes de Rodríguez Saá sí se observa que, a medida que aumenta la IE, hay una mayor proporción que se inclina hacia Macri, aunque en una tendencia menos marcada que el total global.

Cuando observamos la incidencia regional del voto de la primera vuelta en el resultado que obtuvo cada candidato en el balotaje, encontramos que la asociación de los votos de Massa y Macri es pareja en todo el país y que se hace más fuerte en las regiones del norte del país. La región del Noreste, integrada por las provincias de Chaco, Formosa, Corrientes y Misiones, presenta el coeficiente de regresión más alto. No obstante, es en estas provincias donde Scioli obtuvo triunfos importantes. En otras palabras, si bien el Noreste continuó siendo un bastión de Scioli, el trasvase de votos allí favoreció a Macri.

Otro resultado significativo se dio en la provincia de Buenos Aires, donde los votos de Rodríguez Saá presentan un coeficiente cercano al 1,0 en la regresión a favor de $\mathrm{Ma}-$ cri. Es decir, prácticamente todos los votantes de primera vuelta de Rodríguez Saá de la provincia apoyaron a Macri en el balotaje.

Quienes prefirieron a Stolbizer apoyaron en su mayoria a Macri en el balotaje. Este apoyo aumenta en la provincia de Buenos Aires, con una proporción cercana a 0,9, y en la Patagonia, con casi un 0,7. Scioli, por su parte, recibió fuertes apoyos de quienes habían votado a Del Caño, sobre todo en la provincia de Buenos Aires, con una proporción cercana a 0,8, y en la Patagonia, con un 0,7.

Pensando en los factores que movilizaron el voto en el balotaje, esta distribución territorial nos puede mostrar 
la forma en la que la política subnacional puede incidir en los comportamientos electorales a través de las coaliciones electorales que se realizaron de cara a las elecciones provinciales. A modo de ejemplo, Massa realizó alianzas con Cambiemos (Macri) para competir en elecciones a gobernador en provincias del norte como Chaco y Jujuy. Es posible que estas alianzas hayan tenido una influencia fuerte en la posterior decisión de los votantes de Massa ante el balotaje. Otro factor de influencia son las señales que brindaron los candidatos, como los acuerdos de Stolbizer con la gobernadora electa María Eugenia Vidal (Alianza Cambiemos) en la provincia de Buenos Aires, o las expresiones en el mismo sentido de Juan Manuel de la Sota en Córdoba.

Respecto a la competencia ideológica podemos decir que la dimensión espacial izquierda-derecha no parece ser visiblemente predictora del voto en el balotaje. Si esto fuera así, implicaría que los electores elegirían en la segunda vuelta a un candidato más cercano a sus preferencias ideológicas, expresadas en el voto de primera vuelta. En el sistema político argentino esta es de por sí una tarea compleja porque hay otros clivajes que distorsionan la representación (Ostiguy, 2009). Por otra parte, si tenemos en cuenta el comportamiento de los votantes de Massa y Rodríguez Saá (candidatos que provienen del amplio espacio peronista), tampoco la dimensión ideológica peronismo-antiperonismo parece haber adquirido preponderancia en este balotaje para fidelizar el voto.

En los discursos de campaña, Massa parecía estar más cerca de Scioli que del propio Macri, sobre todo en el plano económico. De hecho, Scioli tomó las propuestas nodales de Massa para intentar atraer a sus votantes de cara al balotaje. Sin embargo, esta estrategia no le alcanzó para cosechar la cantidad de nuevas adhesiones necesaria. Algo similar sucedió con los votos de Rodríguez Saá. También los votantes de Stolbizer se inclinaron en mayor medida por Macri a pesar de que, discursivamente, si bien mante- 
nían coincidencias en cuanto a un tipo de administración más institucionalista, mantuvieron diferencias relevantes sobre el modelo económico a aplicar. Los votantes de Del Caño sí parecen haber optado por el candidato más cercano en términos de modelo económico, a pesar de sus profundas diferencias de índole política con Scioli por su raíz peronista.

Entonces ¿cuál es el núcleo de equivalencias de Massa y Rodríguez Saá que motivó que sus electores tuviesen un comportamiento global similar, votando en el balotaje por un candidato que no representaba su raíz de sentido peronista? ¿Cuál es el factor ideológico que decidió al electorado de Stolbizer a votar por Macri a pesar de sus diferencias respecto al modelo económico, por ejemplo? Aquí, en esta coyuntura, emerge una tercera dimensión ideológica que se superpone a las otras dos en la distribución espacial de los comportamientos: el eje kirchnerismo-antikirchnerismo.

A través del eje de campaña "cambio vs. continuidad", Macri pudo sobrevolar de manera menos costosa la complejidad de identidades superpuestas que incidieron en el comportamiento electoral del 2015. Scioli, mientras tanto, no logró instalar un eje de campaña claro para evitar que esa complejidad lo afectase de manera determinante. Por una parte, intentó alejarse un poco del kirchnerismo como forma de acercarse al "votante mediano". ${ }^{16}$ Sin embargo, al mismo tiempo, buscó mantener su diferenciación con Macri para contener el voto propio obtenido en la primera vuelta. En un escenario de marcada polarización ideológica, Macri pudo utilizar consignas generales, dado que su posición era sobreentendida. En ese mismo escenario, Scioli se vio forzado a explicitar posiciones concretas, lo cual redujo sus posibilidades de captar nuevas adhesiones en el balotaje.

${ }^{16}$ Votantes más moderados, indecisos y desideologizados. 


\section{Referencias}

Bárbaro, Julio "Razones de un peronista para votar a Macri". Clarín (11 de noviembre de 2015). http://www.clarin.com/opinion/Kirchnerismo-Disenso-Consenso-Democracia_0_1465653973.html.

Colomer, J. M. (2001) Instituciones politicas. Barcelona: Ariel.

Espinoza, Fernando "Por qué los peronistas votan a Scioli". Clarín (14 de noviembre de 2015b). http://www.clarin.com/opinion/Comunidad_organizada-Movimiento-Liberacion-Dictaduras_0_1467453338.html.

Infobae (18 de noviembre de 2015) "Sergio Massa: 'Le aconsejaría a la gente que no vote en blanco'". http://www.infobae. com/2015/11/18/1770642-sergio-massa-le-aconsejaria-la-gente-que-no-vote-blanco.

La Izquierda Diario (10 de Noviembre de 2015) "Voto en blanco: una campaña en movimiento". http://www.laizquierdadiario. com/Voto-en-blanco-una-campana-en-movimiento.

La Nación (28 de octubre de 2015b) "Sergio Massa afirmó: "Yo no quiero que gane Scioli"". http://www.lanacion.com. ar/1840633-sin-pronunciarse-por-un-candidato-sergio-massa-afirmo-la-argentina-necesita-un-cambio.

(11 de noviembre de 2015c) "Macri consolida su ventaja sobre Scioli en la recta final hacia el ballottage". http://www. lanacion.com.ar/1844379-macri-consolida-su-ventaja-sobrescioli-en-la-recta-final-hacia-el-ballottage.

Labaqui, Ignacio "Segundas vueltas". Berensztein 14 de julio de 2015) http://berensztein.com/segundas-vueltas-por-ignaciolabaqui/.

"¿Se puede dar vuelta un ballotage?" Bastión Digital (26 de octubre de 2015). http://ar.bastiondigital.com/notas/se-puededar-vuelta-un-ballotage.

Lago, I.; J. Montero y M. Torcal (2007) "Introducción: modelos de voto y comportamiento electoral". In Elecciones generales 2004. Madrid : Centro de Investigaciones Sociológicas.

López, Carmen "Las razones económicas del voto por Cambiemos en la región centro". El Cronista Comercial (27 de noviembre de 2015). http://www.cronista.com/3dias/Las-razones- 
economicas-del-voto-por-Cambiemos-en-la-region-centro20151127-0013.html.

Majul, Luis "Scioli y Macri definen por penales" La Nación 18 de julio de 2015). http://www.lanacion.com.ar/1808525-scioli-ymacri-definen-por-penales.

Manguel, Romina "El PRO tiene su limitación, un peronista no puede votar a Macri". INFO News (12 de agosto de 2015). http:// www.infonews.com/nota/241134/el-pro-tiene-su-limitacion -un-peronista.

Ostiguy, P. (octubre de 2009) "Argentina's Double Political Spectrum: Party System, Political Identities, and Strategies", 19442007. Kellogg Institute Working Paper, 361.

Página 12 (18 de noviembre de 2015) "Un guiño de Felipe Solá para Scioli". http://www.pagina 12.com.ar/diario/elpais/1-2863592015-11-18.html.

Quantum GIS Development Team (2015) Quantum GIS Geographic Information System. Open Source Geospatial Foundation Project.: http://qgis.osgeo.org.

R Core Team (2015) R: A Language and Environment for Statistical Computing. Viena: R Foundation for Statistical Computing.

Radio 40 (27 de octubre de 2015). Camino al ballotage "Stolbizer dió un 'sutil' apoyo para 'Cambiemos' y para Macri". http:// laradio40.com.ar/camino-al-ballotage-stolbizer-dio-un-sutilapoyo-para-cambiemos-y-para-macri/.

Ratto, M. y J. Montero (octubre de 2013) "Modelos de voto en Argentina: las elecciones presidenciales de 2007". Postdata, $18(2), 323-364$.

Zacharias, D., D. Sulmont y G. Garibotti (2015) "Elecciones presidenciales Perú 2011: Análisis comparativo de la asociación entre los resultados de la primera y segunda vuelta electoral a nivel mesa de sufragio". Revista Latinoamericana de Opinión Pública,(5), 173-197. 